\title{
Embryo selection strategy in sibling oocytes: A novel approach to measuring the likelihood of single-embryo transfer using a mouse embryo transfer model
}

\author{
FENG-QIN XU, RONG LIU, YING MA, LI LIU, JU-YAN LIANG and YAN MA \\ Center of Reproductive Medicine, Tianjin First Central Hospital, Tianjin 300312, P.R. China
}

Received July 27, 2013; Accepted February 10, 2014

DOI: $10.3892 / \mathrm{mmr} .2014 .1998$

\begin{abstract}
The present study investigated the effect of embryo transfer on post-implantation development using different fertilization approaches in sibling oocyte procedures. C57BL/6, DBA/2, C3H/HeJ and ICR mice were used at 8-10 weeks of age. Mature oocytes were collected, divided into two groups and fertilized using in vitro fertilization (IVF) or intracytoplasmic sperm injection (ICSI). Different numbers of the resulting blastocysts were then transferred into recipients and designated as either: The single embryo transfer (SET) I model (one transferred embryo), the SET II model (six transferred embryos) or the multiple embryo transfer (MET) model (24 transferred embryos). The development efficiency, cell number, number of apoptotic cells in blastocysts, pregnancy efficiency, delivery rate and cumulative pregnancy efficiency were analyzed. IVF-fertilized embryos exhibited higher blastocyst development competence and embryo quality compared with ICSI embryos. The pregnancy and delivery efficiency was not identified to be significantly different between the two SET models, but it was lower in these two models than in the MET model. The cumulative pregnancy efficiency in SET models, calculated using a mathematical equation, was not decreased. In conclusion, embryo quality was shown to be the primary factor in selecting embryos prior to embryo transfer using sibling oocytes. In addition, single blastocyst transfer can be performed in sibling oocytes without compromising cumulative pregnancy rates, independent of the fertilization approach.
\end{abstract}

\section{Introduction}

Assisted reproductive technology (ART) has demonstrated the potential to aid infertile couples to conceive their own offspring since the birth of the first test tube baby in 1978 (1). Since then,

Correspondence to: Dr Feng-Qin Xu or Dr Yan Ma, Center of Reproductive Medicine, Tianjin First Central Hospital, No. 24 Fukang Road, Nankai, Tianjin 300312, P.R. China

E-mail: xufengqin1968@163.com

E-mail: mayan1657@126.com

Key words: assisted reproduction, sibling oocytes, single embryo transfer, multiple embryo transfers novel ART procedures and technologies have been developed to overcome various infertility factors, including in vitro fertilization (IVF) (1), intracytoplasmic sperm injection (ICSI) (2) and preimplantation genetic diagnosis (3). One simple but effective procedure was developed in 1995, and involves a combination of IVF and ICSI using sibling oocytes (4). With this procedure, cases of unexpected total fertilization failure can be avoided. Furthermore, this procedure is beneficial for patients with unexplained infertility (5), mild oligoteratoasthenozoospermia or borderline semen parameters $(6,7)$, severe isolated teratozoospermia (8) and polycystic ovary syndrome with normozoospermic semen $(9,10)$.

Embryo selection is a critical issue in procedures using sibling oocytes. In traditional IVF or ICSI, the embryos used for embryo transfer (ET) are high-quality embryos; however, in sibling oocyte procedures, embryo fertilization approaches should also be considered. At present, the consensus amongst clinicians and embryologists is that IVF embryos should be selected and transferred prior to ICSI embryos with the same or marginally better quality; however, others consider embryo quality to be the gold standard for embryo selection prior to ET.

Previous studies have mainly focused on clinical data analysis. Shveiky et al (11) reported that the development competence of ICSI embryos, including fertilization, pregnancy and implantation efficiency, was not different from that of IVF embryos; Plachot et al (12) reported similar results. Becker et al (13) suggested that a higher number of top quality embryos were obtained following ICSI than following IVF in sibling oocyte procedures, but found that the clinical pregnancy efficiency was similar when IVF (three pregnancies in 11 patients) and ICSI embryos were transferred (eight pregnancies in 24 patients). Staessen et al (10) reported that whether the transferred embryos were from ICSI alone, IVF alone, or a combination of IVF and ICSI did not affect the pregnancy and implantation rates. However, Kihaile et al (14) demonstrated that pregnancy and implantation efficiency is increased when selecting mixed IVF and ICSI embryos together, and in addition, only fertilization efficiency by ICIS approach was superior to IVF-ET. In these clinical studies, different conclusions were suggested based on different infertility symptoms; therefore, it is difficult to be certain of the effects of the fertilization approach on the pregnancy and implantation results.

The other important consideration in selecting embryos for transfer is single embryo transfer (SET), which has been 
utilized in an increasing number of centers. In the USA, the rate of SET tripled from $4.5 \%$ to $14.8 \%$ between 2001 and 2009 (15). Thurin et al (16) performed a double-blind multicenter trial among 11 clinics in Sweden, which found that the birth rates were significantly lower following fresh transfer of one versus two embryos, but the cumulative birth rates were not statistically different between the treatment groups. Gardner et al (17) demonstrated that the ongoing pregnancy rates for SET versus double embryo transfer (DET) were not significantly different in a study evaluating blastocyst transfer. Therefore, SET has been demonstrated to be effective in preventing multiple deliveries without compromising overall pregnancy rates, although it is key in this strategy to select an embryo with higher developmental competence, particularly for sibling oocyte procedures that have two types of embryos from different fertilization approaches. However, there are no relevant studies regarding sibling oocyte procedures; certain studies have focused on candidate genes and comprehensive chromosome screens in traditional IVF or ICSI procedures $(18,19)$.

In the present study, therefore, the pregnancy and delivery outcomes of ICSI alone, IVF alone and a mixture of embryos using mouse SET and multiple embryo transfer (MET) models were compared, and the effect of transferring embryos from different fertilization approaches in sibling oocyte procedures on post-implantation development was investigated.

\section{Materials and methods}

All chemicals were purchased from Sigma-Aldrich (Shanghai, China) unless otherwise indicated.

Ethics statement. The study was approved by the Institutional Review Board at Tianjin First Central Hospital, Tianjin, China. All experiments requiring the use of animals were approved by the Committee on the Ethics of Animal and Medicine of the Peking University Third Hospital, Beijing, China.

Experimental design. In the first experiment, the general characteristics of ICSI and IVF embryos were compared, including pre-implantation development competence, blastocyst cell number and apoptosis analysis. In the second experiment, the pregnancy and delivery outcomes were analyzed with two types of SET models. In the first model, only one blastocyst from IVF or ICSI was transferred into one female recipient. In the second model, six blastocysts from IVF or ICSI were transferred into one recipient since mice are multiparous and normally conceive with $\sim 8-15$ fetuses. In the third experiment, the pregnancy and delivery outcomes were analyzed with the MET model. The blastocysts were classified into five groups: IVF, ICSI, and IVF and ICSI combined following a defined ratio of $1: 1 ; 1: 2$ or 2:1. Twenty-four blastocysts in each group were transferred into one recipient; a total of six recipients were used in each group. In the fourth experiment, the cumulative pregnancy rates in the SET models were calculated and compared with the MET data.

In the IVF experiments, the oocytes were retrieved from C57/BL6 female mice and the sperm were collected from DBA/2 strain male mice; the offspring were thus designated B6D2F1 with a black coat color. In the ICSI experiments, the oocytes were also retrieved from C57/BL6 female mice, but the sperm were collected from $\mathrm{C} 3 \mathrm{H} / \mathrm{HeJ}$ strain male mice; the offspring were designated $\mathrm{B} 6 \mathrm{C} 3 \mathrm{~F} 1$ with an agouti coat color.

Mice. All mice were purchased from the Vital River Company (Beijing, China). The mice used in this study were 8-10 weeks of age, and housed and bred in the Animal Center of the Medical College of Peking University (Beijing, China), according to national legislation for animal care. All mice were maintained individually under controlled temperature and lighting conditions, and provided food and water ad libitum.

C57/BL6 strain female mice (black coat color) were used for oocyte retrieval. The DBA/2 strain male mice (dilute brown coat color) and $\mathrm{C} 3 \mathrm{H} / \mathrm{HeJ}$ strain male mice (agouti coat color) were used as sperm donors. ICR strain female mice (white coat color) were used as ET recipients.

Oocyte collection. Oocytes were collected from the fallopian tubes of adult C57/BL6 strain female mice that were superovulated by injection of $10 \mathrm{IU}$ pregnant mare serum gonadotropins (Hua Fu Biotechnology Company, Tianjin, China) and 10 IU human chorionic gonadotropin (hCG; Hua Fu Biotechnology Company), administered $48 \mathrm{~h}$ apart. Following the retrieval of the oocytes from the oviducts 14-16 h after administration of hCG, the cumulus-oocyte complexes (COCs) were separated into two groups. The COCs from the left oviduct ampulla were digested with $100 \mathrm{U} / \mathrm{ml}$ hyaluronidase, and the naked oocytes were cultured in M16 medium at $37^{\circ} \mathrm{C}$ and $5 \%$ $\mathrm{CO}_{2}$ until ICSI micromanipulation. The COCs from the right oviduct ampulla were used for IVF experiments. The COCs were placed directly into $\mathrm{CZB}$-culture medium at $37^{\circ} \mathrm{C}$ and $5 \% \mathrm{CO}_{2}$ without any treatment, and cultured for $\sim 1 \mathrm{~h}$ prior to IVF manipulation.

$I V F$. Conventional IVF was performed in human tubal fluid (HTF) medium [Vitrolife AB (publ), Göteborg, Sweden], as described in a previous study (20). The sperm were collected from the cauda epididymis of adult DBA/2 strain male mice and capacitated at $37^{\circ} \mathrm{C}$ under $5 \% \mathrm{CO}_{2}$ and $95 \%$ humidity for 1-2 $\mathrm{h}$. The COCs were then moved and co-cultured with motile sperm (final concentration, $1-2 \times 10^{6} / \mathrm{ml}$ ). After $6 \mathrm{~h}$, the COCs were removed from the HTF medium and transferred to the M16 medium (Sigma-Aldrich, St. Louis, MO, USA). Pronuclear formation was considered to represent successful fertilization.

ICSI. ICSI was performed with a $10-\mathrm{mm}$ internal diameter Piezo-driven micropipette (Prime Tech, Ltd., Ibaraki, Japan), as previously described (20). The sperm were collected from the cauda epididymis of adult $\mathrm{C} 3 \mathrm{H} / \mathrm{HeJ}$ strain male mice, and resuspended in Krebs-Ringer bicarbonate solution at a density of $5-10^{6} / \mathrm{ml}$ prior to incubation at $37^{\circ} \mathrm{C}$ with $5 \%$ $\mathrm{CO}_{2}$ in air for $45 \mathrm{~min}$ prior to ICSI. Groups of oocytes were moved from M16 medium to M2 medium (Sigma-Aldrich, St. Louis, MO, USA), and the spermatozoan heads were injected into the oocyte cytoplasm, assisted with a Piezo apparatus (Prime Tech Corp., Tokyo, Japan). The injected oocytes were cultured at $37^{\circ} \mathrm{C}$ under $5 \% \mathrm{CO}_{2}$ and $95 \%$ humidity, and pronuclear formation was considered to represent successful fertilization after $6 \mathrm{~h}$. 
Blastocyst differential staining. The blastocyst cell number was determined, as previously described (20). The blastocysts were transferred to the medium with $0.5 \%$ pronase on day four, and the zona pellucida was removed. The blastocysts were then exposed to rabbit anti-mouse whole serum (Sigma-Aldrich, St. Louis, MO, USA) for $\sim 1 \mathrm{~h}$, and rinsed three times in buffer medium for $5 \mathrm{~min}$. The blastocysts were treated with guinea pig complement (Sigma-Aldrich, St. Louis, MO, USA) and the treated blastocysts were then stained with propidium iodide $(10 \mathrm{mg} / \mathrm{ml})$ and Hoechst $33342(1 \mathrm{mg} / \mathrm{ml})$. Twenty embryos in each group were used for counting.

Apoptosis analysis. Apoptosis was analyzed using a terminal deoxynucleotidyl-transferase-mediated dUTP nick end labeling (TUNEL) apoptosis detection kit (Millipore, Billerica, MA, USA). The blastocysts were fixed in $4 \%$ paraformaldehyde in phosphate-buffered saline (PBS) for $20 \mathrm{~min}$ at $4^{\circ} \mathrm{C}$, then subjected to permeabilization for $20 \mathrm{~min}$ at room temperature with PBS containing 0.2\% Triton X-100. Briefly, the fixed and permeabilized blastocysts were labeled with the TUNEL reaction mixture for $60 \mathrm{~min}$ at $37^{\circ} \mathrm{C}$. The blastocyst nuclei were also stained with DAPI. The results were then analyzed using a Nikon fluorescence microscope (A1R; Nikon, Tokyo, Japan). Twenty embryos in each group were analyzed.

Embryo transfer. The blastocysts were transferred to the uterus of 2.5 day post coitum (dpc) pseudopregnant ICR mice. On day $17.5 \mathrm{dpc}$, the pregnant mice delivered naturally.

Statistical analysis. The results were evaluated using a $\chi^{2}$ test or t-test with SPSS 13.0 software (SPSS, Inc., Chicago, IL, USA) and $\mathrm{P}<0.05$ was considered to indicate a statistically significant difference.

\section{Results}

General characteristics of IVF and ICSI embryos. The pre-implantation development competence was analyzed and compared between IVF and ICSI embryos. The fertilization and cleavage efficiencies in the two groups were not identified to be significantly different at the PN and 2-cell stage $(\mathrm{P}>0.05)$, but the development efficiency of the IVF embryos was significantly higher than the ICSI embryos at the 8-cell and blastocyst stages $(\mathrm{P}<0.05$; Fig. 1A). To assess the embryo quality in the two groups, the blastocyst and apoptotic cell numbers were analyzed by differential staining and TUNEL detection methods, respectively. The results revealed that the inner cell mass (ICM) cell number and the ratio of ICM were higher in the IVF group $(\mathrm{P}<0.05)$, although there was no difference in the trophoderm cell number between the IVF and ICSI groups $(\mathrm{P}>0.05)$. A higher cell apoptosis ratio was observed in ICSI blastocysts ( $\mathrm{P}<0.05$; Fig. 1B).

Pregnancy and delivery outcomes of ICSI and IVF embryos in SET models. In the SET model I, only one blastocyst from IVF or ICSI was transferred into the recipients. The results revealed that $67.5 \%$ of the recipients receiving a single IVF blastocyst became pregnant, which is comparable to single ICSI blastocysts $(60 \%$; P>0.05). On day 17.5 , a healthy neonate was born by natural delivery from each pregnant
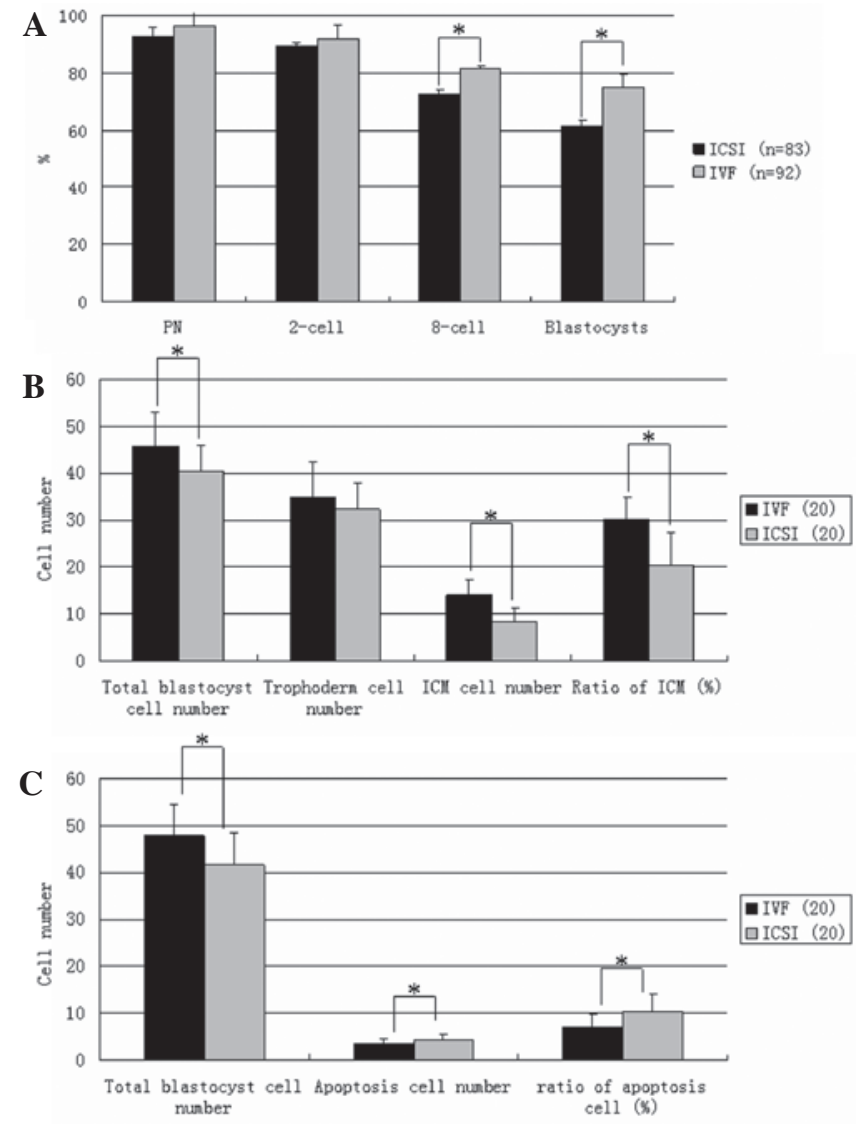

Figure 1. Histogram showing a comparison of pre-implantation development competence and blastocyst quality between in vitro fertilization (IVF) and intracytoplasmic sperm injection (ICSI) embryos. (A) Embryos from the ICSI approach exhibited similar fertilization and cleavage efficiencies, but lower 8-cell and blastocyst development compared with IVF embryos. (B) The blastocyst and inner cell mass (ICM) cell numbers, as well as the ratio of ICM-to-blastocysts was decreased in ICSI blastocysts compared with IVF blastocysts, but the trophoderm cell number did not differ between IVF and ICSI blastocysts. (C) The number of apoptotic cells and the ratio of apoptotic cells-to-total blastocyst cells was increased in ICSI embryos. ${ }^{*} \mathrm{P}<0.05$.

recipient. In ICSI groups, 24 neonates were born from a total 40 blastocysts, and in IVF group, 27 neonates were born from a total 40 blastocysts $(\mathrm{P}>0.05)$. In the SET model II, six blastocysts from IVF or ICSI were transferred into the recipients. The pregnancy efficiency did not differ significantly between the IVF and ICSI groups (83.3 vs. $75.0 \%$; $P>0.05$ ), nor did the delivery rate $(\mathrm{P}>0.05)$ (Table I).

Pregnancy and delivery outcomes of ICSI and IVF embryos in MET models. In an effort to replicate human double or triple ET, 24 blastocysts were transferred into each recipient. Five MET groups were designed as follows: A) 24 Blastocysts from ICSI; B) 16 blastocysts from ICSI and eight blastocysts from IVF; C) 12 blastocysts from ICSI and 12 blastocysts from IVF; D) Eight blastocysts from ICSI and 16 blastocysts from IVF; and E) 24 blastocysts from IVF. All recipients became pregnant following ET in all five groups and the delivery efficiency between groups did not differ. Based on an analysis of neonates from IVF or ICSI blastocysts, mice with a black coat color were B6D2F1 from IVF blastocysts and mice with an agouti coat color were $\mathrm{B} 6 \mathrm{C} 3 \mathrm{~F} 1$ from ICSI blastocysts (Table II). With the exceptions of group A, in 
Table I. The pregnancy and delivery outcomes of IVF and ICSI blastocyst using models SET I and II.

\begin{tabular}{lcccccc}
\hline $\begin{array}{l}\text { SET } \\
\text { model }\end{array}$ & Group & $\begin{array}{c}\text { No. of } \\
\text { recipients }\end{array}$ & $\begin{array}{c}\text { No. of transferred } \\
\text { embryos per recipient }\end{array}$ & $\begin{array}{c}\text { Total no. of } \\
\text { transferred embryos }\end{array}$ & $\begin{array}{c}\text { No. of pregnant } \\
\text { mice (\%) }\end{array}$ & $\begin{array}{c}\text { No. of } \\
\text { neonates (\%) }\end{array}$ \\
\hline I & ICSI & 40 & 1 & 40 & $24(60.0)$ & $24(60.0)$ \\
& IVF & 40 & 1 & 40 & $27(67.5)$ & $27(67.5)$ \\
II & ICSI & 24 & 6 & 144 & $18(75.0)$ & $72(50.0)$ \\
& IVF & 24 & 6 & 144 & $20(83.3)$ & $92(63.9)$ \\
\hline
\end{tabular}

IVF, in vitro fertilization; ICSI, intracytoplasmic sperm injection; SET, single embryo transfer.

Table II. The pregnancy and delivery outcomes of IVF and ICSI blastocyst using MET model.

\begin{tabular}{lccccccc}
\hline Group & $\begin{array}{c}\text { No. of IVF } \\
\text { embryos }\end{array}$ & $\begin{array}{c}\text { No. of ICSI } \\
\text { embryos }\end{array}$ & $\begin{array}{c}\text { No. of } \\
\text { recipients }\end{array}$ & $\begin{array}{c}\text { No. of transferred } \\
\text { embryos per recipient }\end{array}$ & $\begin{array}{c}\text { Total no. of } \\
\text { transferred embryos }\end{array}$ & $\begin{array}{c}\text { No. of pregnant } \\
\text { mice (\%) }\end{array}$ & $\begin{array}{c}\text { No. of } \\
\text { neonates (\%) }\end{array}$ \\
\hline A & 0 & 24 & 6 & 24 & 144 & $6(100.0)$ & $70(48.6)$ \\
B & 8 & 16 & 6 & 24 & 144 & $6(100.0)$ & $59(41.0)$ \\
C & 12 & 12 & 6 & 24 & 144 & $6(100.0)$ & $74(51.4)$ \\
D & 16 & 8 & 6 & 24 & 144 & $6(100.0)$ & $63(43.8)$ \\
E & 24 & 0 & 6 & 24 & 144 & $6(100.0)$ & $71(49.3)$ \\
\hline
\end{tabular}

IVF, in vitro fertilization; ICSI, intracytoplasmic sperm injection; MET, multiple embryo transfer.

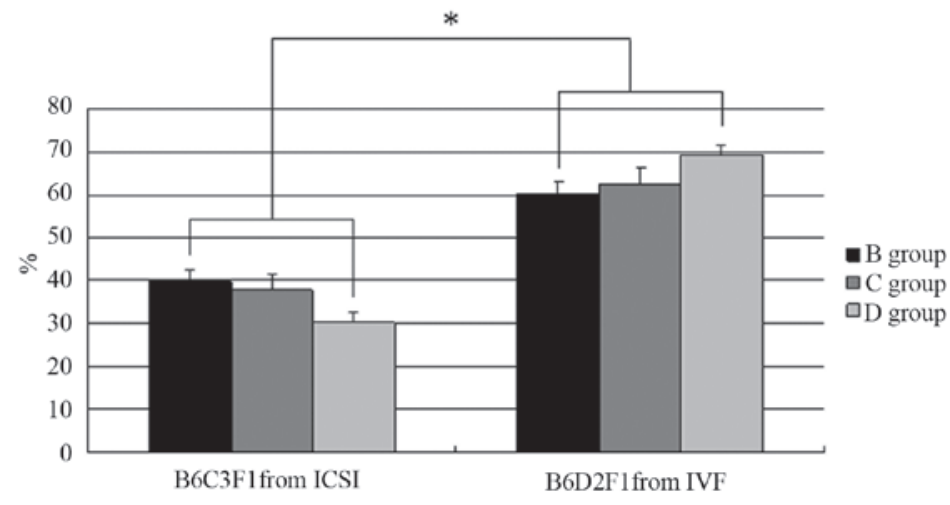

Figure 2. Histogram of the delivery rate of embryos from in vitro fertilization (IVF) or intracytoplasmic sperm injection (ICSI) approaches in the three multiple embryo transfer (MET) mixture groups. Amongst all neonates, the proportion from ICSI blastocysts was significantly lower than that from IVF blastocysts, regardless of the distribution ratio of transferred IVF and ICSI embryos. MET group B, eight IVF and 16 ICSI embryos; MET group C, 12 IVF and 12 ICSI embryos; MET group D, 16 IVF and eight ICSI embryos. " $\mathrm{P}<0.01$.

which ICSI blastocysts alone were transferred, and group E, in which IVF blastocysts alone were transferred, the birth rates of $\mathrm{B} 6 \mathrm{D} 2 \mathrm{~F} 1$ and $\mathrm{B} 6 \mathrm{C} 3 \mathrm{~F} 1$ mice were compared. The results indicated that the delivery rate of ICSI blastocysts was $<40 \%(39.82,37.61$ and $30.32 \%$ in B, C and D groups, respectively). By contrast, the IVF neonatal ratio of all the neonates was $>60 \%(60.18,62.39$ and $69.68 \%$ in B, C and D groups, respectively), which was significantly higher than that of the ICSI counterparts ( $\mathrm{P}<0.01$; Fig. 2$)$.

Cumulative pregnancy efficiency in SET and MET models by mathematical equation. With the establishment of SET and MET models, the cumulative pregnancy efficiency (CPE) was further analyzed using a mathematical equation. If A was designated as pregnancy efficiency in the SET model, the pregnancy probability is A when the mouse is ET for the first time, A x (1-A) for the second time and A x (1-A) ${ }^{2}$ for the third time. When the mouse is ET for $\mathrm{N}$ times, the probability is $\mathrm{A} \times(1-\mathrm{A})^{\mathrm{n}-1}$. Finally, the CPE is the sum of the probability for each ET, and the equation is as follows: $\sum \mathrm{n}=\mathrm{A}+\mathrm{A} \times(1-\mathrm{A})+\mathrm{A} \times(1-\mathrm{A})^{2}+\mathrm{A} \times(1-\mathrm{A})^{\mathrm{n}-1}=1-(1-\mathrm{A})^{\mathrm{n}}$

The equation suggests that the CPE is closely associated with the single pregnancy efficiency and with the ET times (and also indicates the number of available ET embryos). When the two values were higher, the cumulative pregnancy efficiency was $100 \%$. 
To validate the accuracy of the equation, in the present study, the previous data were calculated using this equation. In the ICSI group, the pregnancy rates in MET and SET I and II were 100,75 and $60 \%$, respectively. Thus, if 24 embryos were transferred four times, and six embryos were transferred each time, the CPE was $99.6 \%$. If the embryos were transferred 24 times (using only one embryo each time), the CPE was also 99.9\%. Similar results were obtained when calculating the data in the IVF groups.

\section{Discussion}

One of the key concerns in the sibling oocyte procedure is the embryo selection for ET, as it remains unknown whether the embryo quality or the embryo fertilization method is more important. Moreover, with the wide application of SET in numerous ART centers, this issue is increasingly important in sibling oocyte and other ART procedures.

Using the SET and MET models, the pregnancy and delivery efficiencies on embryo selection in the sibling oocyte procedure were calculated in the present study. The results demonstrate that the pre-implantation development competence and embryo quality were marginally affected by ICSI micromanipulation; however, the ICSI or IVF blastocyst development competence to term was similar in SET models I and II. Furthermore, the IVF, ICSI, 16 ICSI + 8 IVF, 12 ICSI + 12 IVF and 8 ICSI + 16 IVF groups demonstrated similar pregnancy and delivery efficiencies in the MET model, but in the ICSI/ IVF combination groups, IVF blastocysts exhibited greater implantation and delivery competence. Finally, the mathematical equation indicated that the CPE was not any lower in the SET models, and that it was closely associated with the single pregnant efficiency and ET times.

It is well-known that ICSI has been mainly applied to male factor infertility since the first baby was born using this technology in 1992 (2). However, certain studies have indicated potentially harmful effects of ICSI on embryo development and the resulting fetus in human and mouse models $(21,22)$. In the present study, development competence in mouse ICSI embryos was decreased from the beginning of the 8-cell stage. This result was in agreement with previous studies using mice (22), and similar results have been found in humans (23). Cell number and apoptosis are regarded to be better methods to identify blastocyst quality compared with cell morphology (24). Cell apoptosis is regarded as a cellular activity in which the embryos eliminate abnormal cells and prepare for implantation; however, certain studies have indicated that the apoptosis cell number in blastocysts is affected by culture conditions (25-27). In the present study, the ICSI and IVF embryos were cultured in the same culture medium, suggesting that the abnormal cell number was increased by ICSI technology.

To evaluate the post-implantation development competence of ICSI and IVF embryos in sibling oocyte procedures, and to simulate the clinical setting, the SET model I was established, which involves only one embryo, using IVF or ICSI in recipients. However, mice are mammals with multiple gestations, which is different from humans, a generally monotocous mammal. Thus, the SET model II was established, which involves six embryos, with IVF or ICSI transfer to recipients. Normally the litter size of ICR mice is 8-12, thus transferring six embryos is reasonable for the SET model. In a mouse study, the ET number is often $>12$ to ensure pregnancy, but the transferred embryos are usually at the 2-cell stage $(22,28)$. In the current study, the embryos were selected at the blastocyst stage as the development competence of ICSI embryo beyond two cells was decreased compared with the IVF groups. To eliminate potential differences from pre-implantation, the blastocysts were selected in the current study. In the human clinical setting, different clinical outcomes were reported in different centers. Bhattacharya et al (29) summarized and analyzed the data from four UK centers and indicated decreased pregnancy and delivery outcomes when transferring ICSI embryos compared with IVF embryos. However, La Sala et al (30) reported inconsistent results and suggested that ICSI embryos have similar outcomes with IVF embryos following ET in a retrospective analysis. Hourvitz et al (31) also obtained similar results to La Sala et al (30) by analyzing a database from July 2000 and October 2004. The incompatible results may be attributed to multiple factors, including differences between patients, centers, regions and nationalities.

To simulate DET or TET, MET models were established in which 24 blastocysts were transferred to the recipients. Furthermore, to simulate the mixture of ICSI and IVF transferred embryos in the clinical setting, three mixture MET models were established following the possible mixture ratio between IVF and ICSI (1:2, 1:1 and 2:1) in the two groups. The pregnancy rate in each group reached $100 \%$ and the delivery rate between groups was also similar. In a human study, Pandian et al (32) also reported that the likelihood of a live birth in a fresh IVF cycle in SET was significantly decreased; however, certain studies have revealed that there are no significant differences between SET and MET $(33,34)$. Notably, the IVF embryos in the mixture groups exhibited greater implantation and delivery ability than the ICSI counterpart, independent of the number of IVF embryos $(10,15$ or 20 in the three mixture groups), but the ICSI and IVF embryos exhibited similar implantation ability when only one type of embryo was transferred. In the human clinical setting, Shveiky et al (11) reported that the pregnancy and implantation rates in ICSI and IVF embryos did not differ for sibling oocytes. Moreover, Staessen et al (10) concluded that implantation and delivery outcomes were not different when using ICSI, IVF or a mixture of embryos for ET in sibling oocyte procedures. These results were consistent with the findings of the SET and MET models in the present study.

In humans it is almost impossible to distinguish neonates developed from IVF or ICSI embryos, but this issue is easier to resolve in the mouse models using different coat colors for mouse neonates from IVF or ICSI embryos. The present study indicated that IVF embryos exhibited more robust competitiveness in the process of implantation compared with ICSI counterparts in the mixture groups, which could reflect embryo quality. Montag et al (35) reported that successful hatching in vitro is dependent on a sufficiently high number of embryonic cells, which enables the blastocyst expansion and zona shedding that is required prior to implantation. Desai et al (36) suggested that the cell number is one of the key parameters to predict clinical outcome in 
ART. These studies indicate that embryos with a greater cell number have more opportunities to implant into the uterus. In the present study, IVF embryos had a greater cell number in ICM and lesser apoptosis cell number compared with ICSI embryos, which may result in higher competitiveness when combined for ET. In biological evolution, acceptance of superior embryos by the uterus is a possible protective mechanism in the process of reproduction.

In the present study, the pregnancy efficiency was lower in the SET models compared with the MET model; however, the two models exhibited similar CPEs. SET has the advantage of decreasing the multiple pregnancies in ART, and certain clinical studies have shown similar CPE results to those of the current study $(37,38)$. Despite these results, how to select a 'healthy' embryo remains unknown, particularly for sibling oocyte procedures, although a number of studies have focused on screening particular molecular markers in cumulus cells $(39,40)$. Based on the established ET models, a mathematical equation for mouse ET was developed. Predicting pregnancy efficacy in ART has been performed using Kaplan-Meier curves, but rarely by equation (41-44). Lannon et al (45) previously developed an equation in humans using multiple factors to predict pregnancy potential. In the current study, certain extra factors, including freezing/thawing, individual differences, disease factors and the effects of repeated ET on physiology and psychology, were removed from consideration as mouse models were used. The core factors, SET pregnancy rate and ET times (ET times were equal to the available ET number if the SET was applied), were revealed to affect the CPE using the equation developed in the present study. Thus, increasing uterine receptivity by improving ET technology and adjusting female pre-conception physiology (e.g., endometrium blood flow and thickness, and hormone levels), as well as increasing embryo development competence and quality by modifying the culture system, is required prior to improving the CPE.

In conclusion, the present study has provided a criterion for selecting embryos in ART, particularly for sibling oocyte procedures using SET and MET models. Embryo quality was the primary factor while selecting embryos. IVF embryos are more competitive than ICSI embryos in the process of implantation in MET mixture groups, but the fertilization methods did not affect the overall pregnancy and delivery rates. Finally, a mathematical equation demonstrated that the SET pregnancy rate and ET times were key factors with regard to CPE. The present study provides methods to select embryos prior to ET and may aid in promoting the SET application in sibling oocyte procedures.

\section{References}

1. Steptoe PC and Edwards RG: Birth after the reimplantation of a human embryo. Lancet 2: 366, 1978.

2. Palermo G, Joris H, Devroey P and Van Steirteghem AC: Pregnancies after intracytoplasmic injection of single spermatozoon into an oocyte. Lancet 340: 17-18, 1992.

3. Handyside AH, Kontogianni EH, Hardy K and Winston RM: Pregnancies from biopsied human preimplantation embryos sexed by Y-specific DNA amplification. Nature 344: 768-770, 1990.

4. Calderón G, Belil I, Aran B, et al: Intracytoplasmic sperm injection versus conventional in-vitro fertilization: first results. Hum Reprod 10: 2835-2839, 1995.
5. Li Z, Lin H, Xiao W and Wang Y: Fertilization of IVF/ICSI using sibling oocytes from couples with subfertile male or unexplained infertility. J Huazhong Univ Sci Technolog Med Sci 24: 365-368, 2004.

6. Elizur SE, Levron J, Seidman DS, et al: Conventional in vitro fertilization versus intracytoplasmic sperm injection for sibling oocytes in couples with mild oligoteratoasthenozoospermia and couples with normal sperm. Fertil Steril 82: 241-243, 2004

7. van der Westerlaken L, Naaktgeboren $\mathrm{N}$, Verburg $\mathrm{H}$, et al: Conventional in vitro fertilization versus intracytoplasmic sperm injection in patients with borderline semen: a randomized study using sibling oocytes. Fertil Steril 85: 395-400, 2006.

8. Fan W, Li SW, Li L, et al: Outcome of conventional IVF and ICSI on sibling oocytes in the case of isolated teratozoospermia. J Assist Reprod Genet 29: 905-910, 2012.

9. Hwang JL, Seow KM, Lin YH, et al: IVF versus ICSI in sibling oocytes from patients with polycystic ovarian syndrome: a randomized controlled trial. Hum Reprod 20: 1261-1265, 2005.

10. Staessen C, Camus M, Clasen K, et al: Conventional in-vitro fertilization versus intracytoplasmic sperm injection in sibling oocytes from couples with tubal infertility and normozoospermic semen. Hum Reprod 14: 2474-2479, 1999.

11. Shveiky D, Simon A, Gino H, et al: Sibling oocyte submission to IVF and ICSI in unexplained infertility patients: a potential assay for gamete quality. Reprod Biomed Online 12: 371-374, 2006.

12. Plachot M, Belaisch-Allart J, Mayenga JM, et al: Outcome of conventional IVF and ICSI on sibling oocytes in mild male factor infertility. Hum Reprod 17: 362-369, 2002.

13. Becker B, Bertrand E, Van Hoeck J, et al: Outcome of conventional IVF and ICSI on sibling oocytes in patients suffering from teratospermia. Int J Fertil Womens Med 51: 163-169, 2006.

14. Kihaile PE, Misumi J, Hirotsuru K, et al: Comparison of sibling oocyte outcomes after intracytoplasmic sperm injection and in vitro fertilization in severe teratozoospermic patients in the first cycle. Int J Androl 26: 57-62, 2003.

15. Marsh CA, Farr SL, Chang J, et al: Trends and factors associated with the Day 5 embryo transfer, assisted reproductive technology surveillance, USA, 2001-2009. Hum Reprod 27: 2325-2331, 2012.

16. Thurin A, Hausken J, Hillensjö T, et al: Elective single-embryo transfer versus double-embryo transfer in in vitro fertilization. N Engl J Med 351: 2392-2402, 2004.

17. Gardner DK, Surrey E, Minjarez D, et al: Single blastocyst transfer: a prospective randomized trial. Fertil Steril 81: 551-555, 2004.

18. Uyar A, Torrealday S and Seli E: Cumulus and granulosa cell markers of oocyte and embryo quality. Fertil Steril 99: 979-997, 2013.

19. Fragouli E and Wells D: Aneuploidy screening for embryo selection. Semin Reprod Med 30: 289-301, 2012.

20. Yu Y,Ding C, Wang E, et al: Piezo-assisted nuclear transfer affects cloning efficiency and may cause apoptosis. Reproduction 133: 947-954, 2007.

21. Feng C, Wang LQ, Dong MY and Huang HF: Assisted reproductive technology may increase clinical mutation detection in male offspring. Fertil Steril 90: 92-96, 2008.

22. Yu Y, Zhao C, Lv Z, et al: Microinjection manipulation resulted in the increased apoptosis of spermatocytes in testes from intracytoplasmic sperm injection (ICSI) derived mice. PLoS One 6: e22172, 2011.

23. Griffiths TA, Murdoch AP and Herbert M: Embryonic development in vitro is compromised by the ICSI procedure. Hum Reprod 15: 1592-1596, 2000.

24. Van Soom A, Vanroose G and de Kruif A: Blastocyst evaluation by means of differential staining: a practical approach. Reprod Domest Anim 36: 29-35, 2001.

25. Lane $\mathrm{M}$ and Gardner DK: Increase in postimplantation development of cultured mouse embryos by amino acids and induction of fetal retardation and exencephaly by ammonium ions. J Reprod Fertil 102: 305-312, 1994.

26. Cao YJ, Fan XJ, Shen Z, et al: Nitric oxide affects preimplantation embryonic development in a rotating wall vessel bioreactor simulating microgravity. Cell Biol Int 31: 24-29, 2007.

27. Xie Y, Puscheck EE and Rappolee DA: Effects of SAPK/JNK inhibitors on preimplantation mouse embryo development are influenced greatly by the amount of stress induced by the media. Mol Hum Reprod 12: 217-224, 2006.

28. Kimura Y and Yanagimachi R: Intracytoplasmic sperm injection in the mouse. Biol Reprod 52: 709-720, 1995. 
29. Bhattacharya S, Hamilton MP, Shaaban M, et al: Conventional in-vitro fertilisation versus intracytoplasmic sperm injection for the treatment of non-male-factor infertility: a randomised controlled trial. Lancet 357: 2075-2079, 2001.

30. La Sala GB, Nucera G, Gallinelli A, et al: Spontaneous embryonic loss after in vitro fertilization with and without intracytoplasmic sperm injection. Fertil Steril 82: 1536-1539, 2004.

31. Hourvitz A, Lerner-Geva L, Elizur SE, et al: Role of embryo quality in predicting early pregnancy loss following assisted reproductive technology. Reprod Biomed Online 13: 504-509, 2006.

32. Pandian Z, Templeton A, Serour G and Bhattacharya S: Number of embryos for transfer after IVF and ICSI: a Cochrane review. Hum Reprod 20: 2681-2687, 2005.

33. Gerris J: Single-embryo transfer versus multiple-embryo transfer. Reprod Biomed Online 18 (Suppl 2): 63-70, 2009.

34. Veleva Z, Vilska S, Hydén-Granskog C, et al: Elective single embryo transfer in women aged 36-39 years. Hum Reprod 21: 2098-2102, 2006.

35. Montag M, Koll B, Holmes P and van der Ven: Significance of the number of embryonic cells and the state of the zona pellucida for hatching of mouse blastocysts in vitro versus in vivo. Biol Reprod 62: 1738-1744, 2000.

36. Desai NN, Goldstein J, Rowland DY and Goldfarb JM: Morphological evaluation of human embryos and derivation of an embryo quality scoring system specific for day 3 embryos: a preliminary study. Hum Reprod 15: 2190-2196, 2000.

37. Wang YA, Sullivan EA, Healy DL and Black DA: Perinatal outcomes after assisted reproductive technology treatment in Australia and New Zealand: single versus double embryo transfer. Med J Aust 190: 234-237, 2009.
38. Rodríguez Barredo DB, Tur Padro R, Mancini F, Parriego García M, Rodríguez García I, Coroleu Lletget B and Barri Rague PN: Elective single embryo transfer and cumulative pregnancy rate: five-year experience in a Southern European Country. Gynecol Endocrinol 28: 425-428, 2012.

39. Gebhardt KM, Feil DK, Dunning KR, et al: Human cumulus cell gene expression as a biomarker of pregnancy outcome after single embryo transfer. Fertil Steril 96: 47-52, 2011.

40. McKenzie LJ, Pangas SA, Carson SA, et al: Human cumulus granulosa cell gene expression: a predictor of fertilization and embryo selection in women undergoing IVF. Hum Reprod 19: 2869-2874, 2004.

41. Garrido N, Bellver J, Remohí J, et al: Cumulative newborn rates increase with the total number of transferred embryos according to an analysis of 15,792 ovum donation cycles. Fertil Steril 98 : 341-346, 2012

42. Garrido N, Bellver J, Remohí J, et al: Cumulative live-birth rates per total number of embryos needed to reach newborn in consecutive in vitro fertilization (IVF) cycles: a new approach to measuring the likelihood of IVF success. Fertil Steril 96: 40-46, 2011.

43. Luke B, Brown MB, Wantman E, et al: Cumulative birth rates with linked assisted reproductive technology cycles. N Engl J Med 366: 2483-2491, 2012.

44. Malizia BA, Hacker MR, and Penzias AS: Cumulative live-birth rates after in vitro fertilization. N Engl J Med 360: 236-243, 2009.

45. Lannon BM, Choi B, Hacker MR, et al: Predicting personalized multiple birth risks after in vitro fertilization-double embryo transfer. Fertil Steril 98: 69-76, 2012. 